\title{
All-Optical Cryptography through Spectral Amplitude and Delay Encoding
}

\author{
M. L. F. Abbade, L. A. Fossaluzza Jr., C. A. Messani, G. M. Taniguti, E. A. M. Fagotto \\ School of Electrical Engineering, PUC-Campinas, Rod. D. Pedro I- km 136, Campinas, SP, 13086-900, Brazil. \\ abbade@puc-campinas.edu.br. \\ I. E. Fonseca \\ Centro de Informática, Universidade Federal da Paraíba, Cidade Universitária, João Pessoa, PB, 58051-900, \\ Brazil.
}

\begin{abstract}
All-optical data encryption is a promising technology that could lead communication systems to an unprecedented degree of security. In this paper we use computer simulations to systematically investigate a new all-optical cryptography technique that is bit-rate independent and transparent to modulation formats. Encryption encompasses signal spectral slicing followed by two encoding stages that impose different attenuations and delays to each of the spectral slices. We analyze the quality of the encoded signal by evaluating its bit error rate (BER) performance. Results indicate that such BER may achieve 45 and $28 \%$, respectively, for input non-return-to-zero on-off keying (NRZ-OOK) and differential quadrature phase-shift keying (DQPSK) signals. At the receiver side, results suggest that signals may be properly decoded after propagation distances that are compatible with those utilized in metropolitan area networks. The robustness of the technique against waveform analysis and brute force attacks is also approached.
\end{abstract}

Index Terms - Transparent Optical Networks, Optical Cryptography, Spectral Slicing, Spectral Amplitude Encoding.

\section{INTRODUCTION}

Information security is one of the major issues for present communication systems and should be carefully considered in various situations. For instance, lack of communication confidentiality in military, government, and financial sectors could cause enormous damages and severely affect society. Secrecy failures in intra-company communications could lessen the competitiveness and disturb the strategic planning of the involved industry. In incidents where user data are stolen or accessed by unauthorized third parties, service providers not only experience direct financial damage but also face a negative impact on their image.

A common way to minimize data security problems is to encrypt information. This is usually performed in the presentation layer of the Open Systems Interconnection (OSI) model. However, security increases when each of the communications layers implements its own encrypting technology [1]. Presently, most of core networks rely on optical fiber communication systems that are safer than other transmission technologies but are vulnerable to intrusive and non-intrusive fiber tapping [2]. For this reason, recently, security issues and attack management in transparent optical networks 
(TON) became a key point to network operators [3].

In this scenario, various proposals for encryption in the physical and optical layers have been reported in literature [4- 16]. Chaotic communication is a well-known hybrid (electrical and optical) secure communication method [4- 6], where a chaotic carrier is generated through laser oscillation by all-optical [7], or electro optic [8] means, and then the message is embedded in the chaotic carrier to realize data encryption. Several techniques using optical code division multiple access (OCDMA) to protect the confidentiality of user data in access networks are widely analyzed $[9,10]$. Among them, we highlight the relevance of spectral amplitude encoding (SAE) $[11,12]$ and spectral phase encoding (SPE). In special, simulations concerning a single-user OCDMA approach were utilized to investigate how SPE may scramble wavelength-division multiplexing (WDM)-compatible signals [13]. Nonlinear effects and devices have also been extensively considered to deploy all-optical cryptography. In [14], fiber stimulated Brillouin scattering (SBS) effect is used to distort the amplitude and phase of optical signals and to encrypt them. In [15], secure communication schemes based on optical gates, generated by different semiconductor optical amplifier (SOA) nonlinearities, is investigated. Another nonlinear technique utilizes SOA in optical circuits that perform an exclusive-OR operation between a signal and a delayed version of it to enable optical encryption is analyzed in [16].

In this work, we use computer simulations to propose and evaluate an all-optical cryptography [17] technique that, as in those of $[13,16]$, could be applied to signals transmitted through metropolitan WDM-compatible TONs. The technique consists in dividing an input optical signal in spectral slices and in encrypting it in two stages. SAE is deployed in the first stage whereas the second one utilizes delays in the range of bit periods to encrypt the optical signal. The technique is bit-rate independent and transparent to modulation formats. The performance of its encoded and decoded signals is analyzed in terms of their bit error rates (BER). The use of such metric in the presence of attacks based on waveform analysis is discussed and the robustness against brute force attacks is also considered. Finally, propagation performance through metropolitan area TONs is evaluated. To the best of our knowledge, this is the first time in literature that such two-stage cryptography technique is systematically analyzed.

The remaining of this work is organized in the following way. Section II approaches the theoretical features of the considered technique and also some important considerations for the work. The utilized simulation setup is described in Section III. Section IV presents our results concerning the performance of the technique for non-return-to-zero (NRZ) on-off keying (OOK) and differential quadrature phase-shift keying (DQPSK) input signals. Our final discussions and conclusions are presented in Section V.

\section{THEORY AND INITIAL CONSIDERATIONS}

The principle of operation of the considered all-optical cryptography technique may be promptly understood from the block diagram illustrated in Fig. 1. An input optical signal, $s(t)$, enters an optical 
encoder where it is divided into $n$ spectral slices. First, each of these slices receives an attenuation $\alpha_{i}$ ( $i=1$ to $n$ ); this corresponds to SAE. Then, in a second stage of encryption these slices are also submitted to a delay $\tau_{i}$. In frequency domain, the envelope of the signal corresponding to the $i$-th spectral slice may be written as

$$
E_{i}(\omega)=H_{e, i}(\omega) \alpha_{i} e^{-j \omega \tau_{i}} S(\omega)
$$

where $S(\omega)=\int_{-\infty}^{\infty} s(t) e^{-j \omega t} d t$ is the Fourier transform of $s(t)$, and $H_{e, i}(\omega)$ is the transfer function of the optical band-pass filter (OBPF) that generates the the $i$-th spectral slice.

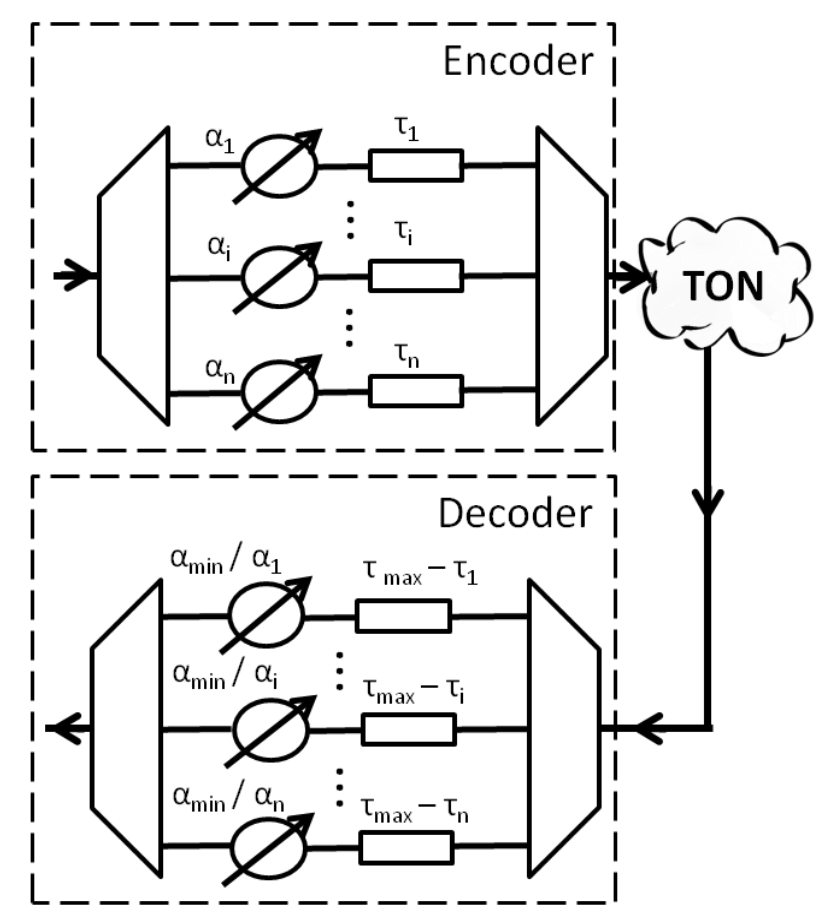

Fig. 1. Block diagram for encoder and decoder.

At the encoder output, an optical coupler or some other similar device is used to merge all slices into a single encoded signal that is a distorted version of the input one. The encoded signal is, then, expressed by

$$
E(\omega)=\sum_{i=1}^{n} E_{i}(\omega)=S(\omega) \sum_{i=1}^{n}\left[H_{e, i}(\omega) \alpha_{i} e^{-j \omega \tau_{i}}\right]
$$

and could be safely transmitted through a TON because, in case it is split and forwarded to an unauthorized route, it should be, at least in principle, unintelligible for any malicious receiver.

Designating the minimum value of coefficient $\alpha_{i}$ by $\alpha_{\min }$ (maximum loss) and the maximum value of $\tau_{i}$ by $\tau_{\max }$, the authorized receiver may recover the transmitted signal by using a decoder which is physically identical to the encoder but that applies attenuations $\alpha_{\min } / \alpha_{i}$ and delays $\tau_{\max } \tau_{i}$ to each spectral slice. Therefore, the decoded signal becomes

$$
D(\omega)=E(\omega) \sum_{i=1}^{n}\left[H_{d, i}(\omega)\left(\alpha_{\min } / \alpha_{i}\right) e^{-j \omega\left(\tau_{\max }-\tau_{i}\right)}\right]
$$

with $H_{d, i}(\omega)$ standing for the transfer function of the OBPF used to recover the the $i$-th spectral slice. 
As in any other optical signal processing technique, the analyzed encoding/ decoding scheme can induce some deterioration to the input signal. In practice, such degradation could arise from distortions imposed by the OBPFs and by interference between signals processed by adjacent spectral slices. To evaluate the influence of such distortions, it is assumed that the input signal is noiseless and it is recalled that its power is its mean square value, $\left\langle s^{2}(t)\right\rangle$, where $\langle x(t)\rangle$ denotes the average of $x(t)$. It is also noted that the distortions generated by the encoding/ decoding processes cause a difference between the decoded and input signals, $n_{d}(t)=d(t)-s(t)$ whose power is given by

$$
\left\langle n_{d}^{2}(t)\right\rangle=\left\langle[d(t)-s(t)]^{2}\right\rangle=\left\langle d^{2}(t)\right\rangle-2\langle d(t) s(t)\rangle+\left\langle s^{2}(t)\right\rangle
$$

where $d(t)=(1 / 2 \pi) \int_{-\infty}^{\infty} D(\omega) e^{j \omega t} d \omega$ is the inverse Fourier transform of $D(\omega)$ and carries the distortions caused by the OBPFs that generate the decoder (Eq. (3)) and encoder (Eqs. (3) and (2)) optical slices. Although, signal distortions are deterministic (Eqs. (3) and (2)), $s(t)$ is random; hence, $n_{d}(t)$ may be treated as a noise created when the input signal $s(t)$ passes by the encoder and by the decoder. Therefore, the optical signal-to-noise ratio, OSNR, caused by such devices is

$$
O S N R=\frac{\left\langle s^{2}(t)\right\rangle}{\left\langle n_{d}{ }^{2}(t)\right\rangle}=\frac{\left\langle s^{2}(t)\right\rangle}{\left\langle d^{2}(t)\right\rangle-2\langle d(t) s(t)\rangle+\left\langle s^{2}(t)\right\rangle},
$$

If the ideal condition $d(t)=s(t)$ is achieved, then the denominator of (5) vanishes and OSNR tends to infinity; this is in agreement with the assumption of noiseless input signal. Lower values of OSNR are associated with higher signal degradations. Since $d(t)$ depends on $s(t)$ (see (3)), the average $\langle s(t) d(t)\rangle$ in (5) may not be considered as the product of the individual averages $\langle d(t)\rangle$ and $\langle s(t)\rangle$.

In this work, we assume that all OBPFs are described by an ideal unitary and rectangular profile in such a way that

$$
H_{d, i}(\omega)=H_{e, i}(\omega)=\operatorname{rect}\left(\frac{\omega-2 \pi f_{c i}}{2 \pi B_{i}}\right)
$$

where $f_{c i}$ and $B_{i}$ are, respectively, the center frequency and bandwidth of the $i$-th spectral slice. It is also considered that $B_{i}$ are contiguously distributed through the essential bandwidth $B_{e}$ of $s(t)$; i.e., there is no superposition and no empty frequency range between any two adjacent spectral slices. With these assumptions and substituting (2) and (6) in (3), it is easy to obtain

$$
D(\omega)=\alpha_{\min } e^{-j \omega \tau_{\max }} S(\omega)
$$

or, in the time domain,

$$
d(t)=\alpha_{\min } s\left(t-\tau_{\max }\right)
$$

This clearly shows that the decoded signal is a delayed and attenuated version of the input optical signal. The cryptographic keys for coding and decoding signals encompass $n, f_{c i}, B_{i}, \alpha_{i}$, and $\tau_{i}(i=1$ to $n)$.

At this point it is important to stress some differences between the technique described above and other work. Conceptually, SAE utilized in the first encoding stage is the same considered for 
application in OCDMA systems. Nevertheless, in the latter approach non-coherent sources with linewidths exceeding $15 \mathrm{~nm}$ are frequently considered [18]. This is not the case in the present work, where we assume that signals externally modulate a laser source with relatively low linewidth, for instance, $90 \mathrm{kHz}$. Therefore, here we deal with signals whose optical bandwidth has the same order of magnitude of its bit rate. Such quantitative change is possible because technology improvements led to the commercial availability of OBPFs with bandwidths as low as $3 \mathrm{GHz}$. On the one hand, in our approach we need to deal with a relatively small number of slices. For example, a 40-GHz bandwidth signal could be divided in at most eight $5-\mathrm{GHz}$ slices. On the other hand, the strategy considered here allows us to encrypt WDM-compatible signals without affecting their bandwidth and channel spacing constraints. Also, since in our approach SAE is not used to provide access to multiple users, as is the case for OCDMA technology, we have no restrictions concerning code orthogonality.

It should also be noted that used delays, $\tau_{i}$, are on the order of the symbol periods of the input signals. Since they are constant in the whole range of frequencies of the spectral slice, each spectral component of the slice acquires its own phase shift. This is an important difference relative to the SPE techniques used in [13], where a constant phase-shift is experienced by all frequency components of a given spectral slice.

Another important issue for this work is the impact of the analyzed technique on the quality of the encoded and decoded signals. The bit error rate (BER) is a good metric for both of them, if the eavesdropper detects the encoded signals with digital receivers. The BER after the decoder, $B E R_{D}$, should be as low as possible and typically accepted values are lower than $10^{-12}$ when no forward error correction (FEC) is used. On the other hand, the BER at the encoder output, $B E R_{E}$, should be as high as possible to prevent signal interpretation by unauthorized receivers. We observe that for binary information the maximum BER value is $50 \%$; this corresponds to the case where a receiver randomly chooses between 0 - and 1- bits.

Although FEC is crucial to allow the transmission of high bit rates optical signals through long distances, it should be carefully used when optical cryptography is considered. This happens because, in principle, FEC could correct bit errors introduced by signals encoders and, thus, favor intruder attacks. Therefore, there is a tradeoff between security and maximum propagation distance in systems with physical-layer encryption. In this work, we assume that a signal is properly encoded when $B E R_{E}$ is $\geq 10 \%$ and consider that utilized FECs are not able to correct BERs higher than $10^{-3}$.

\section{Simulation SETUP}

All simulations considered in this work were performed in a commercially available software program. Since no assumption concerning $S(\omega)$ is made, (1)- (8) should work properly for any modulation format and any bit rate. To illustrate the first of these properties, we consider simulations regarding NRZ-OOK and DQPSK signals. As it is interesting to compare the performance of both of these modulation formats, we decided to use a bit rate, $R_{b}$, that could be feasible for both of them. 
This led to the choice for $R_{b}=40 \mathrm{Gbps}$ because, at this bit rate, DQPSK is a modulation standard and the electronics for implementing NRZ-OOK is not so complex. Obviously, the technique should work for other higher and lower bit rates, provided that OBPFs with appropriate bandwidth and spectral profiles are available. It should be noted that the optical spectrum of the considered 40 Gbps NRZOOK and DQPSK signals occupy bandwidths of, respectively, 80 and $40 \mathrm{GHz}$. Therefore, they fit, respectively, to the $100-$ and $50-\mathrm{GHz}$ ITU grids. Such diversity is interesting to show that the technique investigated in this paper applies for both of such grids.

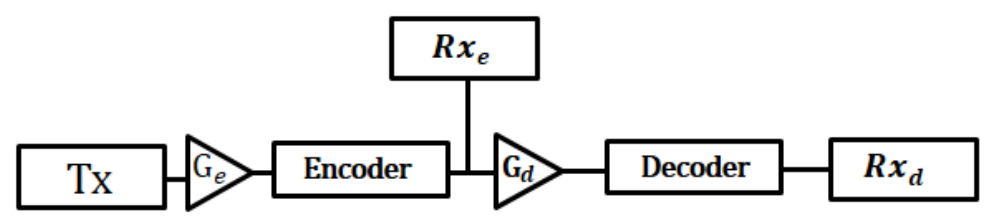

(a)

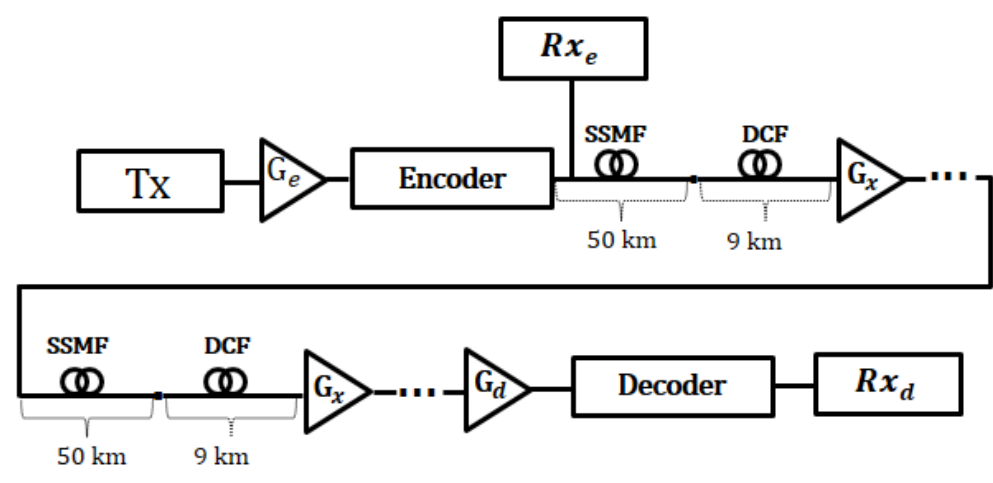

(b)

Fig. 2. Setups for (a) back-to-back and (b) propagation simulations.

Simulations concerning back-to-back and propagation of the encoded signal were considered. The setup utilized for the back-to-back case is illustrated in Fig. 2(a). In a transmitter, Tx, a 2048 pseudorandom bit sequence (PRBS) is used to modulate an optical carrier at frequency $f_{c}=193.1 \mathrm{THz}$ with either NRZ-OOK or DQPSK modulation schemes. The peak power of this optical signal is set to 0 $\mathrm{dBm}$. A first optical amplifier boosts this signal that is then driven to the all-optical encoder. The encoder acts accordingly to the description of Section II. At the output of such device, the signal power is kept at $0 \mathrm{dBm}$ thanks to gain $G_{e}$ provided by the first optical amplifier. The BER performance of this encoded signal is statistically evaluated after it is passed by a receiver, $\mathrm{Rx}_{\mathrm{e}}$. Direct and coherent detection are, respectively, assumed for NRZ-OOK and DQPSK signals. A second amplifier boosts the encoded signal by a gain $G_{d}$. A decoder, as the one presented in Fig. 1, is utilized to decode the optical signal which is finally detected by receiver $\mathrm{Rx}_{\mathrm{d}}$. Gain $G_{d}$ is also set to provide an optical power of $0 \mathrm{dBm}$ at the input of $\mathrm{Rx}_{\mathrm{d}}$.

To simulate TON propagation, the encoded signal is also transmitted through a set of $1050-\mathrm{km}$ long standard single-mode fibers (SSMF), as illustrated in Fig. 2(b). SSMF 16 ps/(nm.km) chromatic 
dispersion is balanced out by 7.1-km long dispersion compensating fibers (DCF) with dispersion of $90 \mathrm{ps} /(\mathrm{km} . \mathrm{nm})$ at $193.1 \mathrm{THz}$. SSMF and DCF attenuations are, respectively, of 0.20 and $0.60 \mathrm{~dB} / \mathrm{km}$ and fiber losses are evened out by optical amplifiers of gain $G_{x}$ placed at the end of each optical link. In this way the peak power of the encoded signal remains at $0 \mathrm{dBm}$ at the output of every optical link. The noise figure of all optical amplifiers used in these simulations is of 5.0 dB. The optical signal-tonoise ratio (OSNR) at the fiber input is $45 \mathrm{~dB}$.

As in the back-to-back situation, at the receiver side, another optical amplifier, with gain $G_{d}$, boosts the encoded signal in such a way that the peak power at the output of the decoder is also set to $0 \mathrm{dBm}$. The encoded signal is then passed through the all-optical decoder where it is decrypted and then photo-detected. The decoder OBPFs features are the same as those of the encoder. Once the optical power at the input of the photo-detectors is always the same, we are able to make a fair comparison between the bit error rates of the encoded and decoded signals.

\section{RESULTS AND DisCUSSIONS}

In this section, we present the results concerning the performance of the considered technique. Initially, to investigate the influence of slice attenuation and delay on $B E R_{E}$ and $B E R_{D}$ and to deal with a tractable number of parameters, only three spectral slices are considered (subsections A, B, and C). After some insights are provided by such initial analysis, the robustness of the technique against some possible attacks (subsections D and E) and its propagation performance (subsection F) are evaluated by considering seven spectral slices.

\section{A. NRZ-OOK Signals: Spectral and Time Domain Analysis}

Fig. 3 illustrates the optical spectra of an input NRZ-OOK signal at the (a) encoder input, (b) encoder output, and (c) decoder output for the back-to-back case (no TON between the encoder and decoder) with $n=3, \alpha_{1}=5 \mathrm{~dB}, \alpha_{2}=20 \mathrm{~dB}$, and $\alpha_{3}=0 \mathrm{~dB}$ e $\tau_{1}=\tau_{2}=\tau_{3}=0$ ps. Fig. 3(b) evidences the presence of three spectral slices $40 \mathrm{GHz}$. Abrupt spectral changes in the interface between spectral slices are a consequence of the considered rectangular filter profiles; such filters are also responsible for the suppression of spectral components outside the range between 193.04 and $193.16 \mathrm{THz}\left(f_{c^{-}}\right.$ $3 R_{b} / 2$ and $f_{c}-3 R_{b} / 2$ ). This suppression is also observed for the decoded signal spectrum shown in Fig. 3(c). However, since the amplitude of the suppressed spectral components is relatively low, its influence on the decoded signal is small; in fact, our simulation results indicate that $B E R_{D}$ is lower than $10^{-15}$ in this situation. 

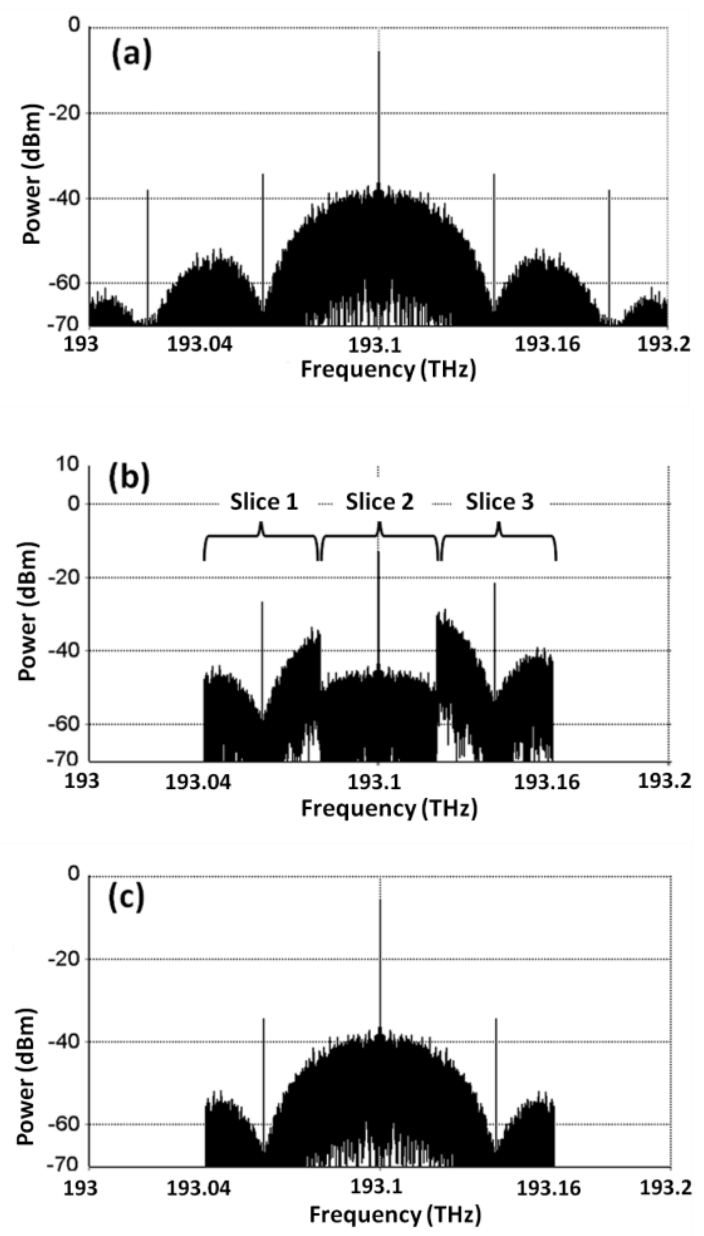

Fig. 3. Optical spectra of an input NRZ-OOK signal at the (a) encoder input, (b) encoder output, and (c) decoder output for the back-to-back case. Encoding parameters are $n=3, \alpha_{1}=5, \alpha_{2}=20$ and $\alpha_{3}=0 \mathrm{~dB}$ and $\tau_{1}=\tau_{2}=\tau_{3}=0$ ps.

It is also important to investigate the technique in time-domain. For this sake, Fig. 4 illustrates a 21bit sequence extracted from the considered 2048 PRBS for the signals at the (a) encoder input, (b) encoder output, and (c) decoder output for the back-to-back case; eye-diagrams, for the 2048 PRBS, are shown as an inset and attenuation and delay parameters are the same as before. Fig. 4(b) shows that the encoded signal is a distorted version of the input one; in fact, such encoded signal resembles an analog one and its eye-diagram is completely closed. It should be noted that eventually a space in the input signal appears as a signal with relatively high power in the encoded signal; also, a mark in the input signal occasionally is observed as a low power signal in its encoded version. This is a consequence of pulse spreading that is theoretically expected, as suggested by the inverse Fourier Transform of (2)

$$
e(t)=s(t) * \sum_{i=1}^{n}\left[\alpha_{i} h_{e, i}\left(t-\tau_{i}\right)\right]
$$

where $h_{e, i}(t)$ is the inverse Fourier Transform of $H_{e, i}(\omega)$ and '*' represents the convolution operation between two signals $x(t)$ and $y(t), x(t) * y(t)=\int_{-\infty}^{\infty} x(t) y(t-\tau) d \tau$. In fact, since $\alpha_{1}$ and $\alpha_{2}$ are different from unity, the aforementioned pulse spreading presented in Fig. 4(b) is a result of a destructive 
interference among the $\alpha_{i} h_{e, i}(t)$ terms in the summation of (9). A good theoretical background for this discussion is found at [19].

As indicated by the bit sequence on the top of Figs. 4(a) and (b), if the encoded sequence were detected six out of 21 bits would be in error (29\% of errors). This is in rather good agreement with $B E R_{E}=33.6 \%$ estimated by our simulation software for the 2048 PRBS. Fig. 4(c) suggests that the decoded signal is very similar to the input one. A small ripple is a consequence of the previously discussed suppression of some spectral components. In fact, for the results of Fig. $4,\left\langle s^{2}(t)\right\rangle=1.8912$, $\left\langle d^{2}(t)\right\rangle=1.8897$, and $\langle s(t) d(t)\rangle=1.8890$ (all in arbitrary units); this leads to an OSNR of $\sim 28 \mathrm{~dB}$. Such high value agrees with the fact that the decoded signal is considerably similar to the input one.
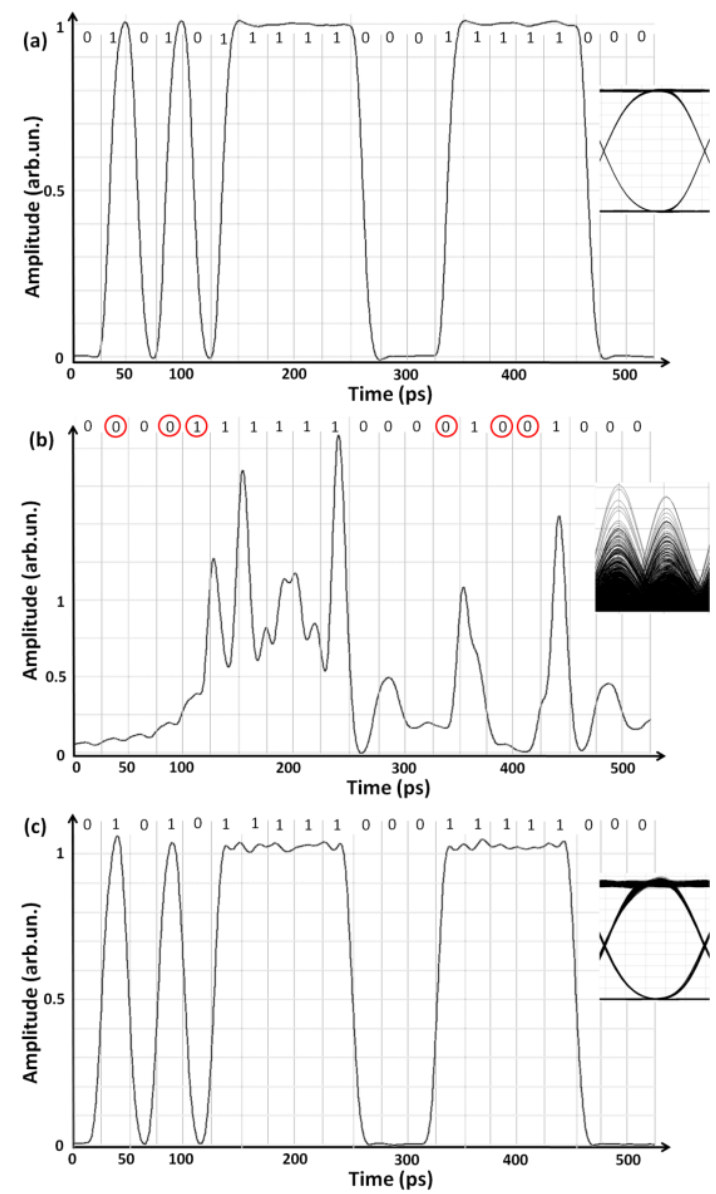

Fig. 4. An NRZ-OOK sequence for the signals at the (a) encoder input, (b) encoder output, and (c) decoder output for the back-to-back case. Encoding parameters are $n=3, \alpha_{1}=5, \alpha_{2}=20$ and $\alpha_{3}=0 \mathrm{~dB}$ and $\tau_{1}=\tau_{2}=\tau_{3}=0$ ps.

\section{B. NRZ-OOK Signals: Influence of Spectral Slices Attenuation and Delay on the Bit Error Rate of Encoded and Decoded Signals.}

Considering $n=3$, Fig. 5 illustrates the BER of the encoded signal as a function of $\alpha_{2}$ and for i) $\alpha_{1}=$ $\alpha_{3}=0 \mathrm{~dB}$; ii) $\alpha_{1}=0 \mathrm{~dB}$ and $\alpha_{3}=5 \mathrm{~dB}$, and iii) $\alpha_{1}=5 \mathrm{~dB}$ and $\alpha_{3}=0 \mathrm{~dB}$. As $\alpha_{2}$ is increased, $B E R_{E}$ also increases. Moreover, for $\alpha_{2}>20 \mathrm{~dB}, B E R_{E}$ acquires an asymptotical behavior with a maximum value of $\sim 45 \%$. This is in quite good agreement with the maximum BER value of $50 \%$ pointed out in Section II. It is observed that the curves corresponding to cases ii) and iii) are closely superimposed; 
this happens because of the symmetry between the spectral slices with $i=1$ and $i=3$. In all of these cases the BER of the decoded signals were below $10^{-15}$, which suggest an appropriate recovering of the input signal.

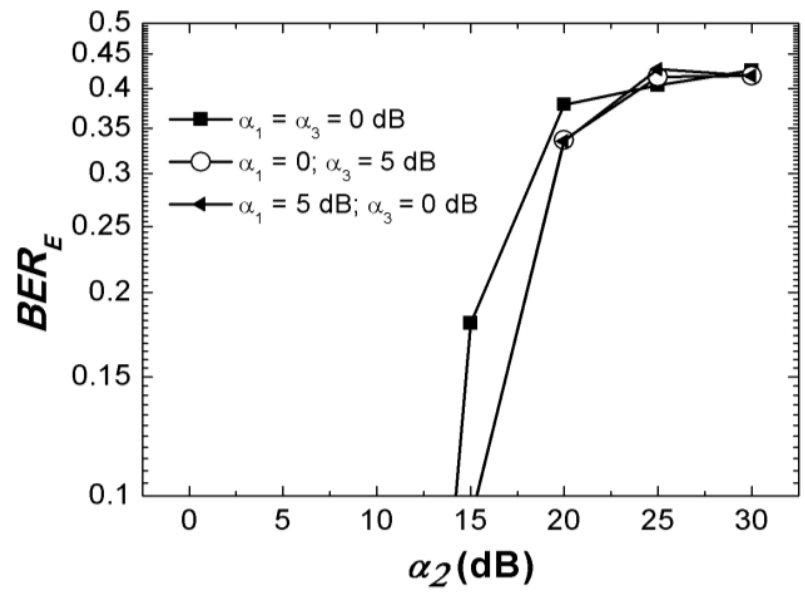

Fig. 5. Dependence between the BER of an encoded NRZ-OOK signal and spectral slice attenuations. Other encoding parameters are $n=3$ and $\tau_{1}=\tau_{2}=\tau_{3}=0$ ps.

Figure 6 exhibits the influence of delays applied to the BER of the encoded signal when $\alpha_{1}=\alpha_{2}=$ $\alpha_{3}=0 \mathrm{~dB}$. As before, the $n=3$ spectral slicing situation is considered. The $i$-th spectral slice delay is varied in increments of bit duration, $T_{b}$. Generally speaking, $B E R_{E}$ increases when $\tau_{i}$ also increases. Thanks to the spectral symmetry of the considered case, variations on $\tau_{1}$ and $\tau_{3}$ are equivalent in terms of their impact on $B E R_{E}$. Moreover, since the amplitude of the central spectral slice $(i=2)$ is higher than those of the other two ones, $B E R_{E}$ is mostly affected by variations on $\tau_{2}$. However, even for $\tau_{2}$ variations, $B E R_{E}$ is at most of $\sim 0.2 \%$, which is well below our considered limit of $10 \%$. This suggests that delays exert a relatively lower influence on $B E R_{E}$ and that they should not be used as a single cryptographic key parameter to provide good quality (high BER) to encoded signals. The BER of the decoded signal for all the cases considered in Fig. 6 was, again, inferior to $10^{-15}$ and indicates the good quality of the technique in back-to-back situations.

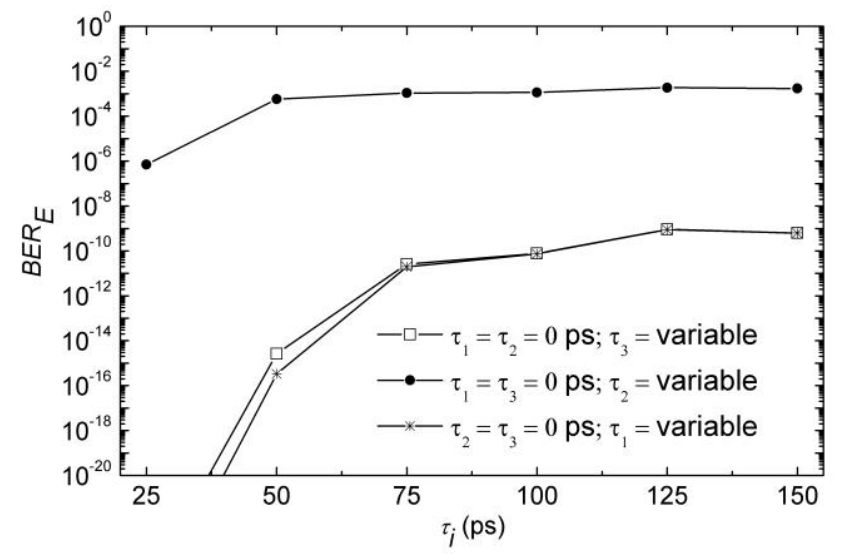

Fig. 6. Dependence between the BER of an encoded NRZ-OOK signal and spectral slice delays. Other encoding parameters are $n=3$ and $\alpha_{1}=\alpha_{2}=\alpha_{3}=0 \mathrm{~dB}$. 
We also consider the case where delays are applied in combination to attenuations to the spectral slices. For $n=3$, Fig. 7 shows that $B E R_{E^{\sim}} 0.01 \%$ for $\tau_{2}=0$, but that it becomes higher than $20 \%$ for some values of $\tau_{2}$. In contrast to the results presented in Fig. 6, this indicates that delays do impose a meaningful increment to $B E R_{E}$ when they are applied in conjunction to attenuations to the spectral slices of the encoded signal. Fig. 7 also shows there is no simple trend between $B E R_{E}$ and $\tau_{2}$. At first sight, this may not seem an attractive point because it may require a very good matching between the delays utilized in the encoder and in the decoder. However, the absence of tendency between both of these variables also improves the security of the cryptographic key and could be used as an advantageous issue. Results discussed in this paragraph illustrate the effectiveness of simultaneously attenuating and delaying spectral slices for signal encryption purposes.

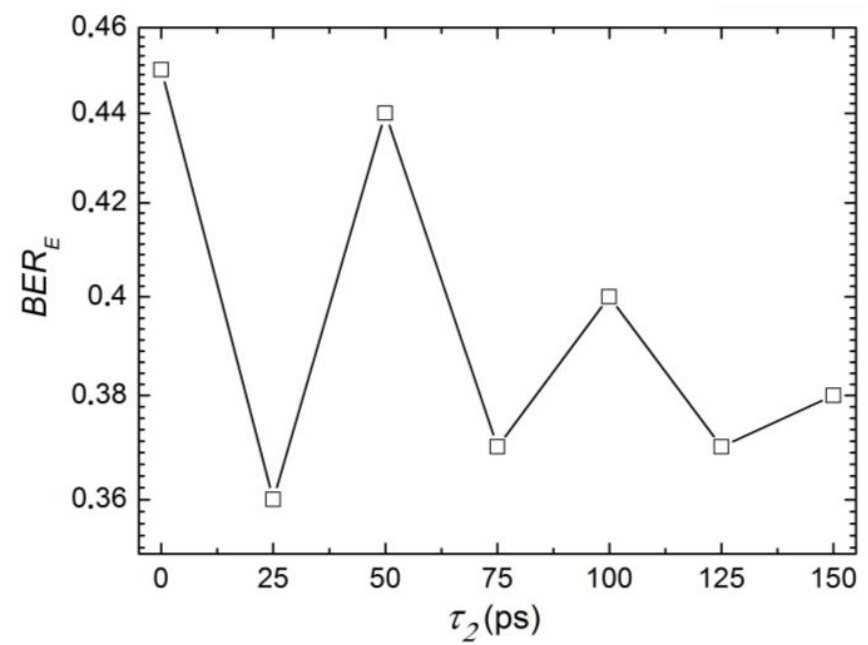

Fig. 7. Relation between the BER of an NRZ-OOK encoded signal and the delay of the central slice. Other encoding parameters are $n=3, \alpha_{1}=5, \mathrm{~dB} \alpha_{2}=20 \mathrm{~dB}, \alpha_{3}=0 \mathrm{~dB}$, and $\tau_{1}=\tau_{3}=0 \mathrm{ps}$.

\section{DQPSK Signals}

To illustrate the effect of the technique on multi-level phase-modulated signals, we plot on Fig. 8 the optical spectra of 40 Gbps DQPSK signals at the (a) encoder input, (b) encoder output, and (c) decoder output for the back-to-back cases. All parameters are the same utilized to obtain the results presented in Fig. 3 with exception of the optical band-pass filters utilized to generate the optical slices whose bandwidths are now of $20 \mathrm{GHz}$. This is necessary because DQPSK signals convey the double of bits per symbol than NRZ-OOK ones and so their bandwidth are halved. In accordance to this, Fig. 8(a) illustrates that the discrete spectral components are spaced from the carrier one by integer multiples of $20 \mathrm{GHz}$. As in the case of NRZ-OOK signals, the distortion of the encoded signal and the spectral suppression of spectral components outside the range of the considered slices are also verified in Figs. 8(b) and 8(c). 

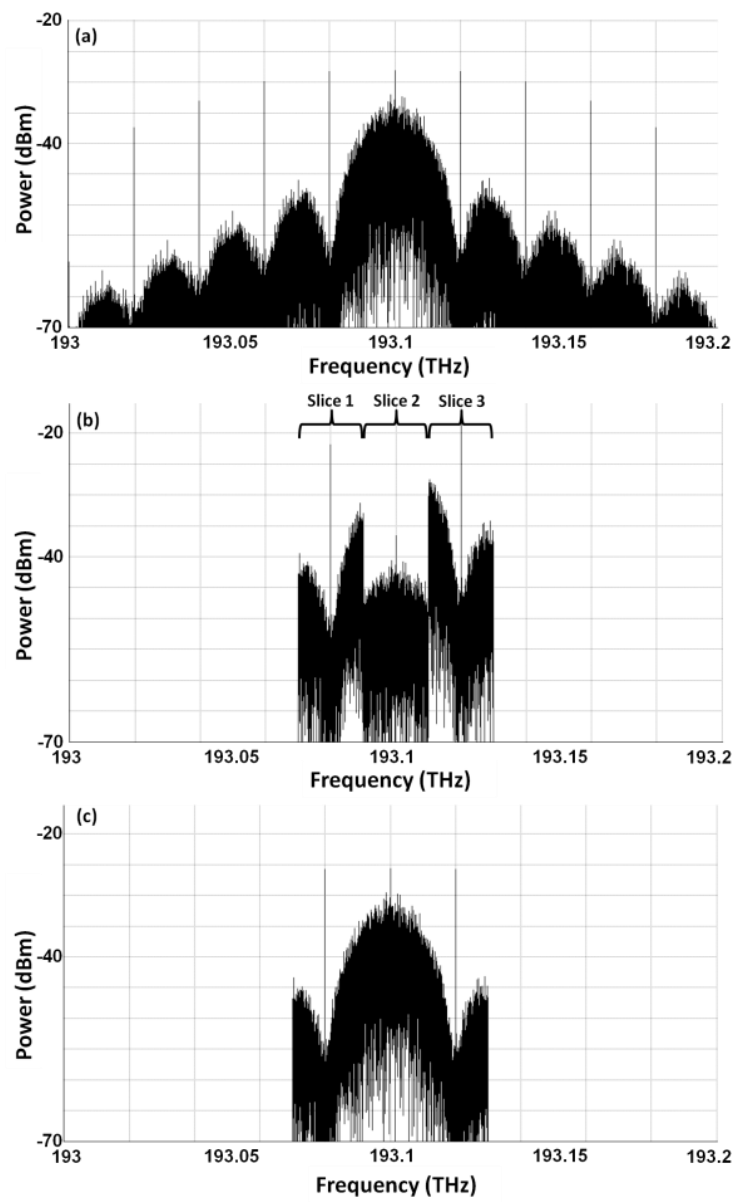

Fig. 8. Optical spectra of an input DQPSK signal at the (a) encoder input, (b) encoder output, and (c) decoder output for the back-to-back case. Encoding parameters are $n=3, \alpha_{1}=5, \alpha_{2}=20$ and $\alpha_{3}=0 \mathrm{~dB}$ and $\tau_{1}=\tau_{2}=\tau_{3}=0 \mathrm{ps}$.

In the time domain, the in-phase and quadrature-phase components of the input, encoded and decoded DQPSK signals also present the same characteristics as those shown in Fig. 4 for the NRZOOK signals. For this only reason, results concerning such temporal analysis are not shown here. The BER of the decoded signal is again lower than $10^{-15}$. Moreover, results indicate that $\left\langle s^{2}(t)\right\rangle=1.2164$, $\left\langle d^{2}(t)\right\rangle=1.2183\langle s(t) d(t)\rangle=1.2169$ (all in arbitrary units); hence, OSNR is $\sim 31 \mathrm{~dB}$ that, again, agrees with the fact that the decoded signal is very similar to the input one.

Fig. 9(a) presents the trend between $B E R_{E}$ and $\alpha_{2}$ for $n=3$. As for the NRZ-OOK case, whose curves are reproduced in Fig. 9(b), $B E R_{E}$ grows monotonically when $\alpha_{2}$ is augmented. However, the maximum value of $B E R_{E}$ is now around $28 \%$ and happens when one of the side slices $(i=1$ or $i=3)$ is also attenuated. The higher $B E R_{E}$ maximum value for NRZ-OOK signals, $\sim 45 \%$, is a consequence that amplitude-modulated signals are more affected by attenuation distortions (caused by SAE) than phase-modulated signals.

The relation between $B E R_{E}$ and $\tau_{i}$ for $n=3$ is depicted in Fig. 10(a). Differently from the NRZ case, $B E R_{E}$ often assumes values higher than $10 \%$. In addition to such difference, increasing $\tau_{i}$ may either cause $B E R_{E}$ to rise or to drop, which has the pros and cons previously discussed. Both of these dissimilarities with the NRZ situation are possibly explained by recalling the fact that time delays 
impose signal phase changes. Therefore, the influence of such delays on phase-shifted signals is expected to be much more effective than in amplitude-shifted ones. To facilitate a comparison between the DQPSK and NRZ-OOK situations, Fig. 9(b) reproduces the curves plotted in Fig. 5.
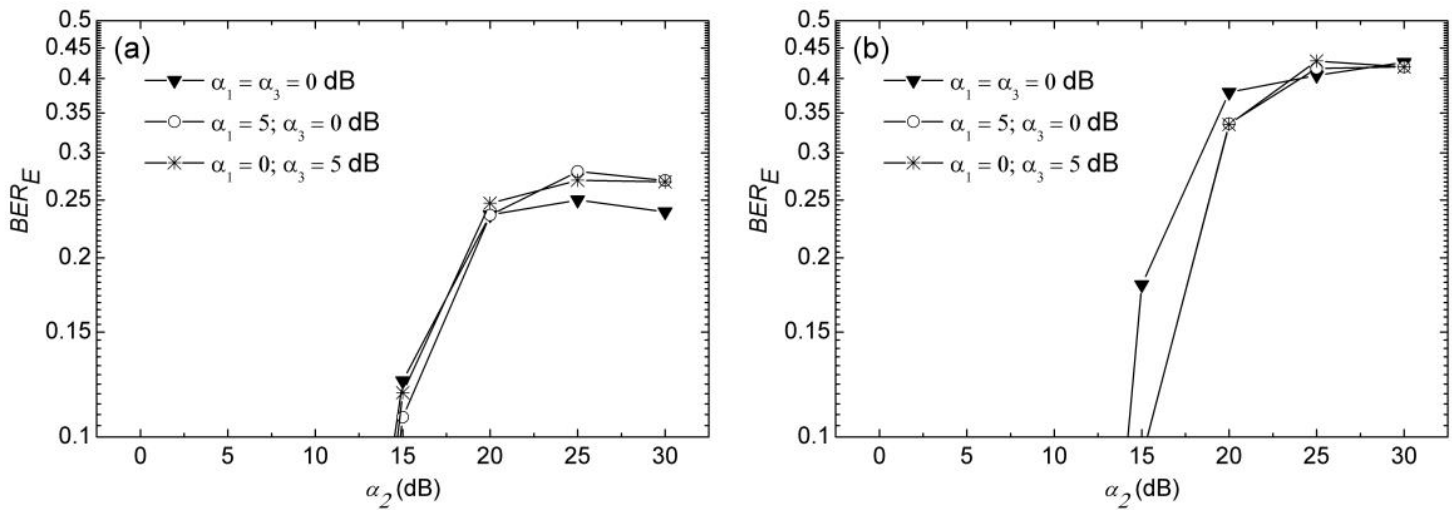

Fig. 9. Dependence between the BER of (a) an encoded DQPSK signal and (b) an encoded NRZ-OOK signal and spectral slice attenuations. Other encoding parameters are $n=3$ and $\tau_{l}=\tau_{2}=\tau_{3}=0 \mathrm{ps}$
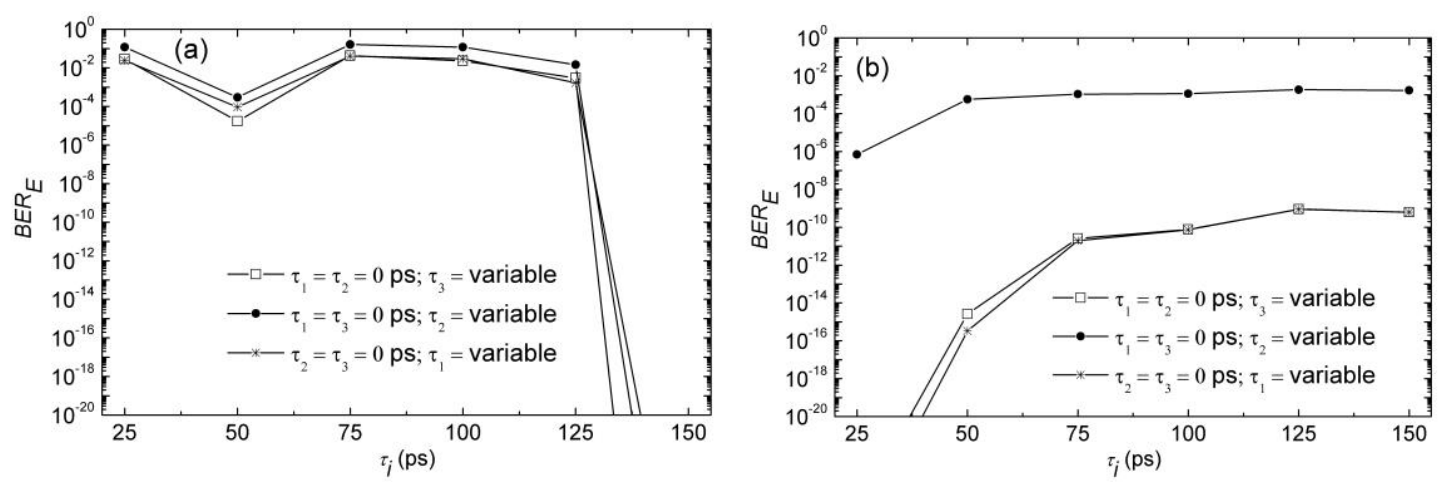

Fig. 10. Dependences between the BER of (a) an encoded DQPSK signal and (b) an encoded NRZ-OOK signal and spectral slice delays. Other encoding parameters are $n=3$ and $\alpha_{1}=\alpha_{2}=\alpha_{3}=0 \mathrm{~dB}$. (b)

The graph of Fig. 11(a) shows the performance of $B E R_{E}$ when a delay in the central slice $(i=2)$ is applied in combination to attenuations to the spectral slices $\left(n=3, \alpha_{l}=5, \mathrm{~dB} \alpha_{2}=20 \mathrm{~dB}, \alpha_{3}=0 \mathrm{~dB}\right.$, and $\tau_{l}=\tau_{3}=0 \mathrm{ps}$ ). From $\tau_{2}=0$ to $25 \mathrm{ps}, B E R_{E}$ is increased from $\sim 20 \%$ to $\sim 32 \%$. At higher values of $\tau_{2}$, $B E R_{E}$ tends to an asymptotical value of $\sim 28 \%$. As indicated in Fig. 11(b), which is a reproduction of Fig. 7, such tendency also happens, for higher values of $\tau_{2}$, in the NRZ-OOK situation. Physically, this asymptotical behavior means that, after a given critical delay value, $\tau_{c}$, signal becomes so distorted that the bit error rate of the encoded signal cannot be further enhanced. The critical delay is shorter for DQPSK signals, because, as stated before, phase-shifted signals are more affected by delay variations than amplitude-shifted ones. In despite of this asymptotical convergence for $B E R_{E}$, it is shown in subsection IV.F that there are important benefits in utilizing high values of $\tau_{i}$. 

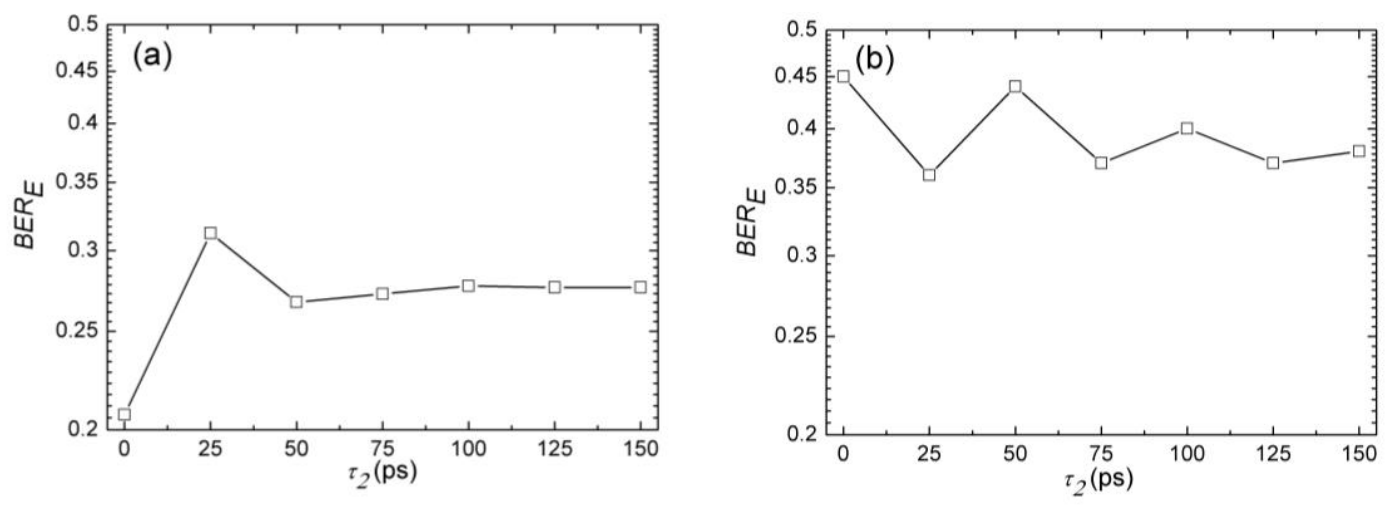

Fig. 11. Relations between the BER of (a) DQPSK and (b) NRZ-OOK encoded signals and the delay of the central slice. Other encoding parameters are $n=3, \alpha_{1}=5, \mathrm{~dB} \alpha_{2}=20 \mathrm{~dB}, \alpha_{3}=0 \mathrm{~dB}$, and $\tau_{1}=\tau_{3}=0 \mathrm{ps}$.

\section{Propagation}

It is important to evaluate the performance of signals after propagation through a TON. Fig. 12 shows the $B E R_{D}$ performance of NRZ-OOK signals when the propagation scenario described in Section III (Fig. 2b) is taken into account for five different optical powers and for $B_{e}=$ a) $60 \mathrm{GHz}$ and b) $120 \mathrm{GHz}$. Encoding parameters are $n=7, \alpha_{1}=8 \mathrm{~dB}, \alpha_{2}=6 \mathrm{~dB}, \alpha_{3}=0 \mathrm{~dB}, \alpha_{4}=1 \mathrm{~dB}, \alpha_{5}=1 \mathrm{~dB}, \alpha_{6}=$ $5 \mathrm{~dB}$, and $\alpha_{7}=10 \mathrm{~dB}, \tau_{l}=3 T_{b}, \tau_{2}=7 T_{b}, \tau_{3}=8 T_{b}, \tau_{4}=10 T_{b}, \tau_{5}=8 T_{b}, \tau_{6}=6 T_{b}$, and $\tau_{7}=7 T_{b}$. It is considered that polarization mode dispersion (PMD) is compensated by digital signal processing (DSP) elements at the receiver side. In fact, such compensation is necessary even if uncoded signals are propagated. For the $60 \mathrm{GHz}$ case, distances of up to $400 \mathrm{~km}$ are possible $\left(B E R_{D} \leq 10^{-12}\right)$ for -3 and $-6 \mathrm{dBm}$. Higher powers lead to signal degradation possibly associated to self-phase modulation (SPM). For $B_{e}=120$ $\mathrm{GHz}$, the maximum reach is around $350 \mathrm{~km}$ and happens when -3 and $0 \mathrm{dBm}$ are used.

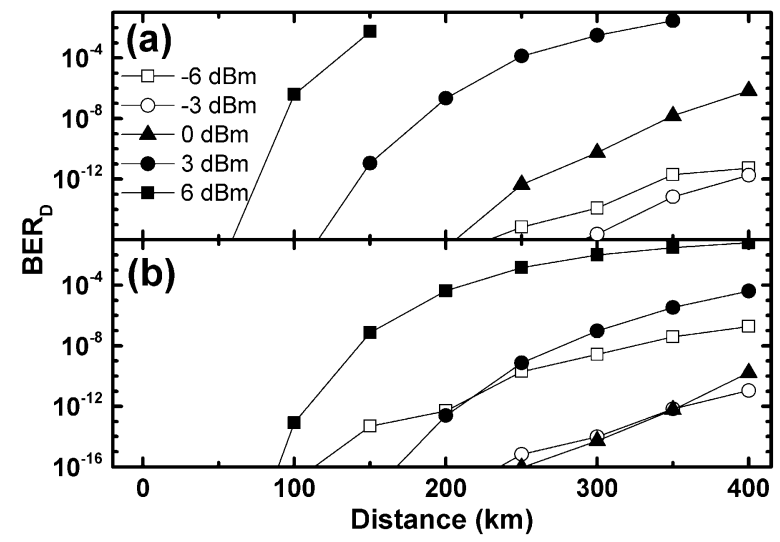

Fig. 12. BER of the decoded NRZ-OOK signals with effective bandwidth of (a) 60 and (b) $120 \mathrm{GHz}$ as a function of propagation distance for different optical powers.

A similar analysis is performed for DQPSK signals with the following encoding parameters $n=7$, $\alpha_{1}=8 \mathrm{~dB}, \alpha_{2}=6 \mathrm{~dB}, \alpha_{3}=0 \mathrm{~dB}, \alpha_{4}=1 \mathrm{~dB}, \alpha_{5}=1 \mathrm{~dB}, \alpha_{6}=5 \mathrm{~dB}$, and $\alpha_{7}=10 \mathrm{~dB}, \tau_{1}=3 T_{s}, \tau_{2}=7 T_{s}, \tau_{3}=$ $8 T_{s}, \tau_{4}=10 T_{s}, \tau_{5}=8 T_{s}, \tau_{6}=6 T_{s}$, and $\tau_{7}=7 T_{s}$, where $T_{s}$ is the symbol period. Results are shown in Fig. 13 where it is observed that the higher the optical power is, the higher $B E R_{D}$ becomes. This is again a manifestation of SPM degradation. Assuming a FEC able to correct BERs lower than $10^{-3}$ is used, it is 
easy to verify that signals with $B_{e}=30$ and $60 \mathrm{GHz}$ lead, respectively, to propagation distances of 400 and $300 \mathrm{~km}$ for optical powers of -3 and $-6 \mathrm{dBm}$. It is interesting to note that for both modulation formats, the use of a lower $B_{e}$ caused a better propagation distance performance. Also, in all the considered cases, propagation distances are compatible with those expected for metropolitan area networks.

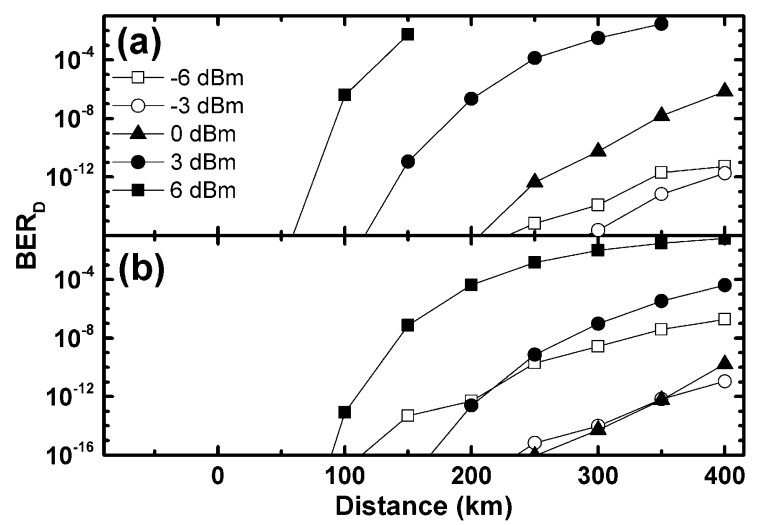

Fig. 13. BER of the decoded DQPSK signals with effective bandwidth of (a) 30 and (b) $60 \mathrm{GHz}$ as a function of propagation distance for different optical powers.

It should be noted that in practice the shape of the filters will not be ideal as considered in (4). Therefore, (6) will no longer be valid and some pulse distortion is expected for the decoded signal. This could be solved by optimizing the OBPF frequency spacing [20] or by using decoder filters with the reciprocal profile of those utilized in the encoder, i.e. $H_{e, i}(\omega)=1 / H_{d, i}(\omega)$ in the spectral range of interest.

\section{E. Robustness to Input Bit Patterns}

As a signal is propagated through a TON, it may be intercepted by an intruder who attempts to decode it. Two techniques for achieving such goal are approached in this subsection and in the next one. Here, the resilience of the technique to input bit patterns (IBP) is considered. Subsection F discusses the technique robustness to brute force attacks.

Fig. 14 illustrates an encoded signal when the following IBPs are repeated five times and utilized to modulate an NRZ-OOK input signal: a) 10, b) 110, c) 1110, and d) 11110; $n=7$, attenuations $\alpha_{I}=8$ $\mathrm{dB}, \alpha_{2}=6 \mathrm{~dB}, \alpha_{3}=0 \mathrm{~dB}, \alpha_{4}=1 \mathrm{~dB}, \alpha_{5}=1 \mathrm{~dB}, \alpha_{6}=5 \mathrm{~dB}$, and $\alpha_{7}=10 \mathrm{~dB}$ and $\tau_{i}=0$ (i=1 to 7) were utilized. To investigate the performance of the cryptographic technique for different bandwidths, we consider the cases where the effective bandwidths of the signals are $B_{e}=3 R_{b}=120 \mathrm{GHz}$ and $B_{e}=$ $3 R_{b} / 2=60 \mathrm{GHz}$. In both of them, an output bit pattern (OBP) is always observed. In fact, even when a PRBS is used it is possible to find some kind of output pattern; i.e. the encoded signal that corresponds to, for example, the input bit sequence 111 is approximately the same no matter the position of such sequence in the PRBS. An intruder could use this fact to promote an attack and try to discover the encoding cryptographic key. After sending a given input bit pattern, such malicious user 
could utilize a scope to save the encoded signal waveform and to perform off-line calculations to determine the set of considered attenuations.
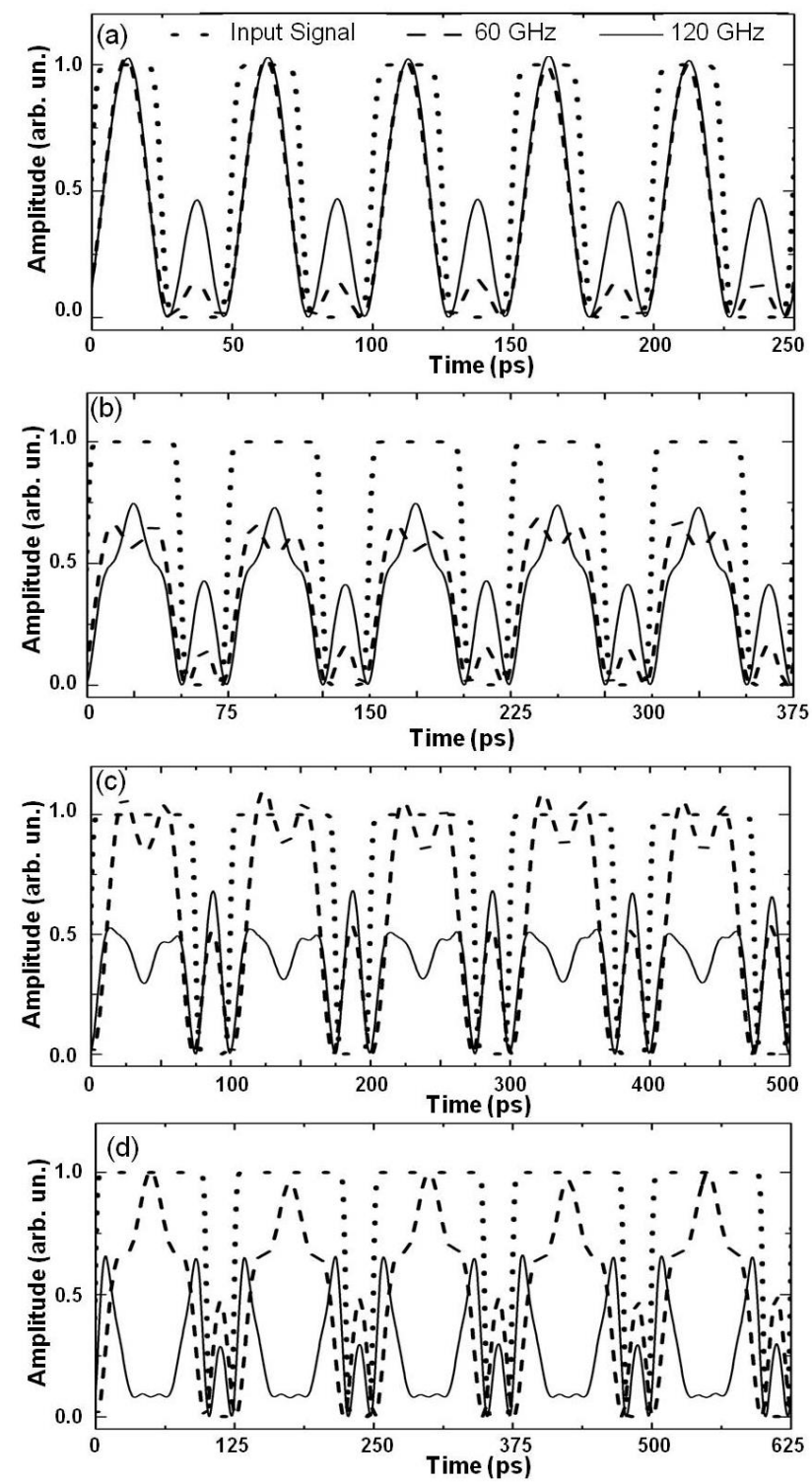

Fig. 14. Input NRZ-OOK signals for bit patterns a) 10, b) 110, c) 1110, and d) 11110 repeated five times and their correspondent output signals with effective bandwidths of 60 and $120 \mathrm{GHz}$. Encoding parameters are: $n=7, \alpha_{1}=8 \mathrm{~dB}$, $\alpha_{2}=6 \mathrm{~dB}, \alpha_{3}=0 \mathrm{~dB}, \alpha_{4}=1 \mathrm{~dB}, \alpha_{5}=1 \mathrm{~dB}, \alpha_{6}=5 \mathrm{~dB}$, and $\alpha_{7}=10 \mathrm{~dB}$ and $\tau_{i}=0$ ( $i=1$ to 7$)$.

OBP is broken when delays are included in the cryptographic key. Figs. 15 (a), (b), and (c) show this important feature for the same parameters considered in the results presented in Fig. 12, but with $\tau_{1}=3 T_{b}, \tau_{2}=7 T_{b}, \tau_{3}=8 T_{b}, \tau_{4}=10 T_{b}, \tau_{5}=8 T_{b}, \tau_{6}=6 T_{b}$, and $\tau_{7}=7 T_{b}$. The maximum value of $\tau_{i}$ is, thus, $\tau_{\max }=10 T_{b}$. The absence of an OBP is a consequence of temporal scrambling caused by such delays, which, indeed corresponds to an artificially introduced dispersion. It is interesting to note that, unlike in fiber and other practical optical materials, this dispersion does not vary monotonically with frequency because each spectral slice receives its own delay. 

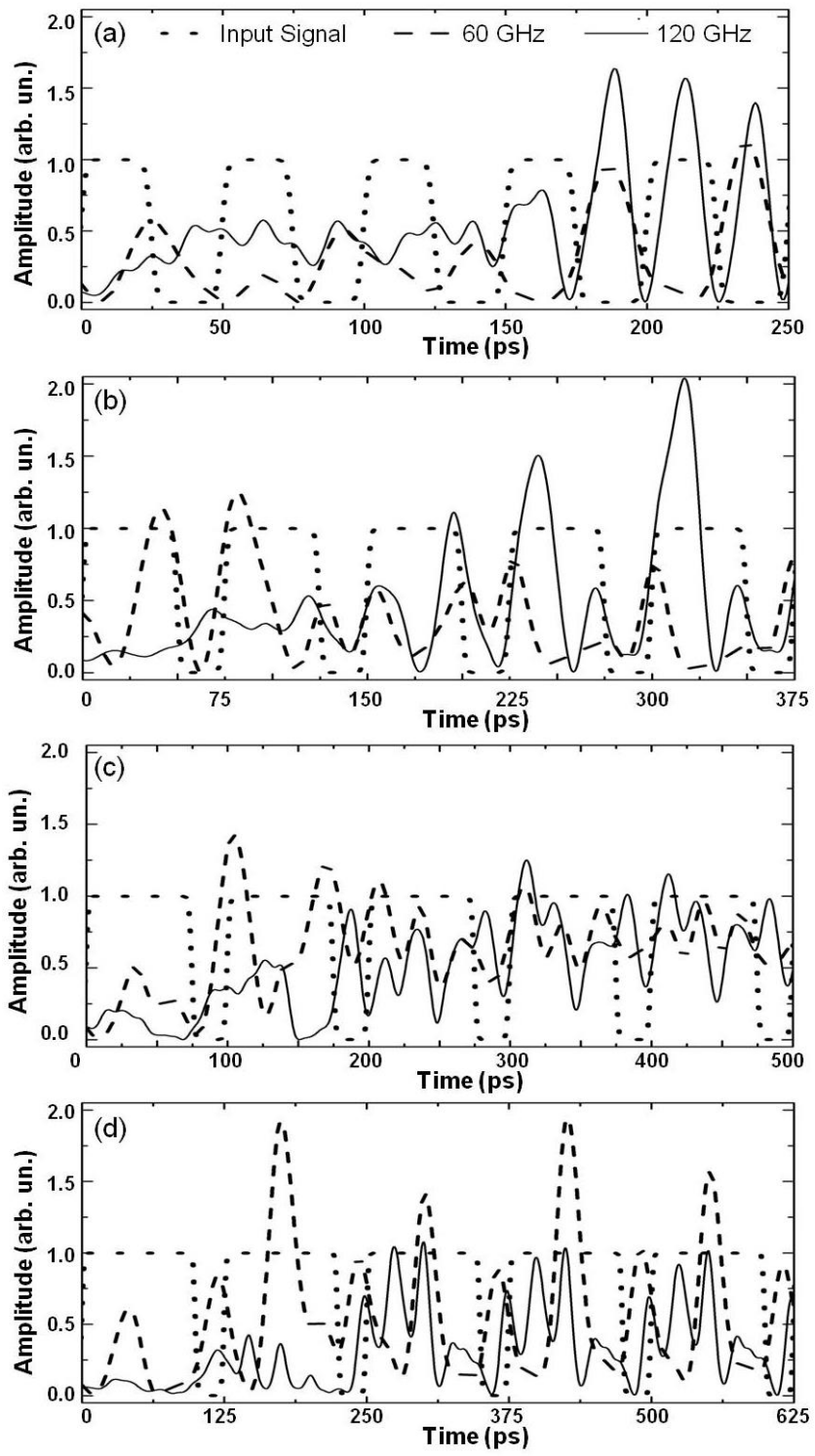

Fig. 15. Input NRZ-OOK signals for bit patterns a) 10, b) 110, c) 1110, and d) 11110 repeated five times and their correspondent output signals with effective bandwidths of 60 and $120 \mathrm{GHz}$. Encoding parameters are: $n=7, \alpha_{I}=8 \mathrm{~dB}$, $\alpha_{2}=6 \mathrm{~dB}, \alpha_{3}=0 \mathrm{~dB}, \alpha_{4}=1 \mathrm{~dB}, \alpha_{5}=1 \mathrm{~dB}, \alpha_{6}=5 \mathrm{~dB}$, and $\alpha_{7}=10 \mathrm{~dB}, \tau_{1}=3 T_{b}, \tau_{2}=7 T_{b}, \tau_{3}=8 T_{b}, \tau_{4}=10 T_{b}, \tau_{5}=8 T_{b}, \tau_{6}=$ $6 T_{b}$, and $\tau_{7}=7 T_{b}$.

However, if the IBP is repeated during time intervals longer than the maximum value of $\tau_{i}$, then the OBP reappears. This is shown in Fig. 15(d), after the second repetition of the 5-bits input sequence 11110. Hence $\tau_{\max }$ could be evaluated after an attack with a properly long IBP sequence.

It is important to note that, in practice, this kind of attack could be avoided. For example, most of TON client equipment utilizes time division multiplexing (TDM) technology to send its user data. Therefore, if the eavesdropper is one of these users, his data is interleaved with those of other users and he cannot send a very long IBP. In this case, $\tau_{\max }$ should be set to be larger than the duration of the longest sequence allowed for a user. If, on the other hand, a TON client transmits its user data in frames that do not use TDM, a different cryptographic key could be utilized for each frame. In this 
way, the eavesdropper could discover the value of $\tau_{\max }$ used to encrypt his own data, but not of other users.

Figs. 16 and 17 show the influence of IBP for an input DQPSK signal, respectively, with and without the application of delays to the spectral slices. Attenuations are $\alpha_{1}=8 \mathrm{~dB}, \alpha_{2}=6 \mathrm{~dB}, \alpha_{3}=0$ $\mathrm{dB}, \alpha_{4}=1 \mathrm{~dB}, \alpha_{5}=1 \mathrm{~dB}, \alpha_{6}=5 \mathrm{~dB}$, and $\alpha_{7}=10 \mathrm{~dB}$ and, only for Fig. 17, delays are $\tau_{1}=3 T_{s}, \tau_{2}=7 T_{s}$, $\tau_{3}=8 T_{s}, \tau_{4}=10 T_{s}, \tau_{5}=8 T_{s}, \tau_{6}=6 T_{s}$, and $\tau_{7}=7 T_{s}$, where $T_{s}$ is the symbol period. The considered bandwidths are $B_{e}=60$ and $30 \mathrm{GHz}$. Behaviors and conclusions are the same as in the case for NRZOOK input signals. It is interesting to note that we performed simulations concerning the technique reported in [13] and found that, as in the case of SAE analyzed here, SPE also leads to OBP.
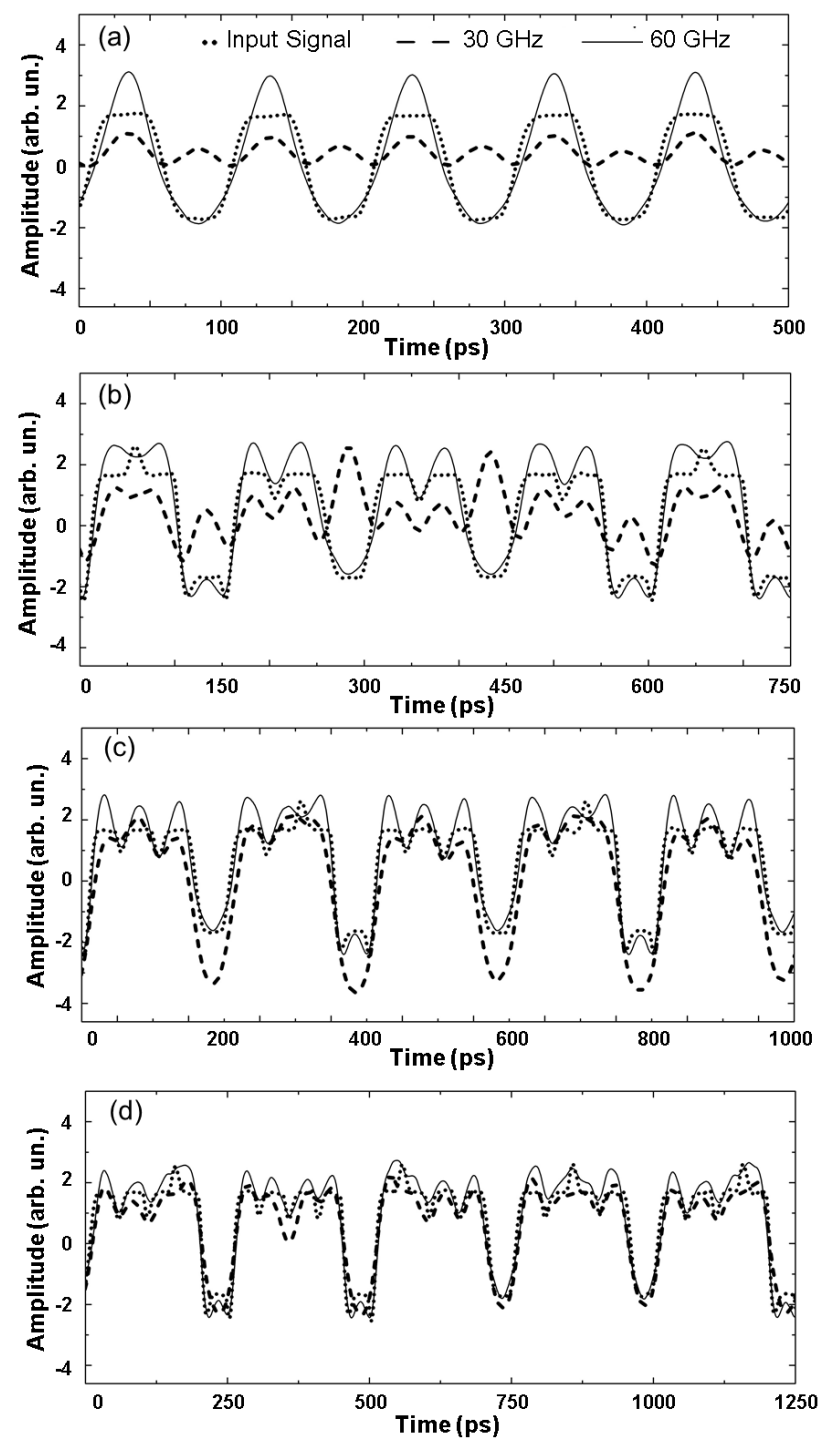

Fig. 16. Input DQPSK signals for bit patterns a) 10, b) 110, c) 1110, and d) 11110 repeated five times and their correspondent output signals with effective bandwidths of 30 and $60 \mathrm{GHz}$. Encoding parameters are: $n=7, \alpha_{1}=8 \mathrm{~dB}$, $\alpha_{2}=6 \mathrm{~dB}, \alpha_{3}=0 \mathrm{~dB}, \alpha_{4}=1 \mathrm{~dB}, \alpha_{5}=1 \mathrm{~dB}, \alpha_{6}=5 \mathrm{~dB}$, and $\alpha_{7}=10 \mathrm{~dB}$ and $\tau_{i}=0$ ( $i=1$ to 7$)$. 

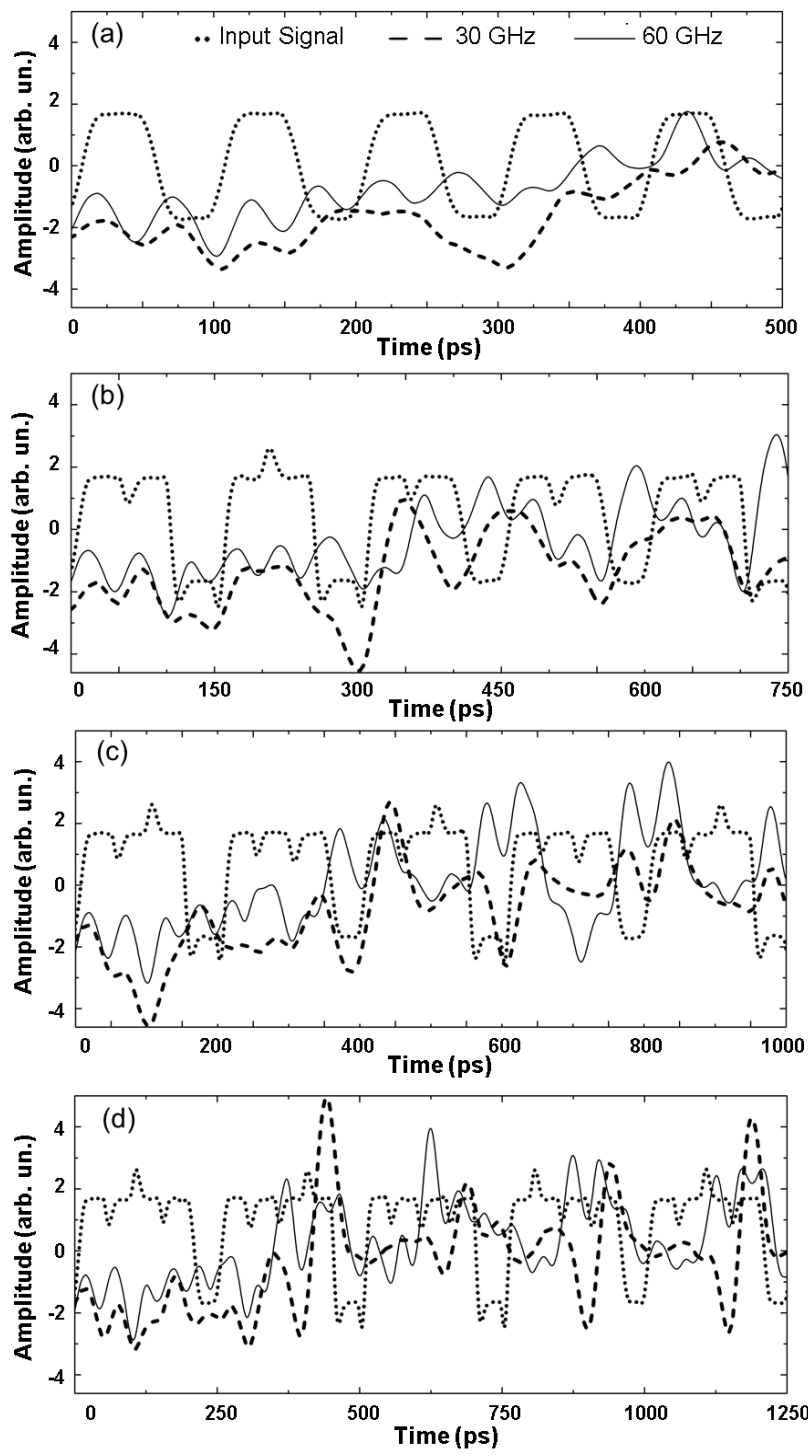

Fig. 17. Input DQPSK signals for bit patterns a) 10, b) 110, c) 1110, and d) 11110 repeated five times and their correspondent output signals with effective bandwidths of 30 and $60 \mathrm{GHz}$. Encoding parameters are: $n=7, \alpha_{l}=8 \mathrm{~dB}$, $\alpha_{2}=6 \mathrm{~dB}, \alpha_{3}=0 \mathrm{~dB}, \alpha_{4}=1 \mathrm{~dB}, \alpha_{5}=1 \mathrm{~dB}, \alpha_{6}=5 \mathrm{~dB}$, and $\alpha_{7}=10 \mathrm{~dB}, \tau_{l}=3 T_{s}, \tau_{2}=7 T_{s}, \tau_{3}=8 T_{s}, \tau_{4}=10 T_{s}, \tau_{5}=8 T_{s}, \tau_{6}=6 T_{s}$, and $\tau_{7}=7 T_{s}$.

$B E R_{E}$ is a good metric for evaluating the quality of the encoded signal when digital receivers are used by unauthorized users. However, the discussion presented in this subsection shows that under waveform attacks, such metric is not enough to describe the capacity of eavesdroppers decoding signals. In this case, the degree of signal scrambling should also be taken into account.

\section{F. Robustness to Brute Force Attacks}

In brute force attacks, the eavesdropper systematically varies the parameters of the cryptographic key in order to decode the encrypted signal. Here, we consider an example of such attack by supposing that the eavesdropper knows the position, the bandwidth and the attenuations of the cryptographic key. Therefore, he should discover only the values of $\tau_{i}$. Encoding parameters are the 
same as those for the results presented in Fig. 15. Only the NRZ-OOK case is considered in this subsection and it is assumed that the attack is well succeeded if $B E R_{D} \leq 10^{-12}$ for a FEC-less situation.

Table I illustrates the values of $B E R_{D}$ for some combinations of $\tau_{i}$. It is seen that the attack becomes successful when, at least, the delays of the three central spectral slices $\left(\tau_{3}, \tau_{4}\right.$ and $\left.\tau_{5}\right)$ are precisely known.

TABLE I. BER OF THE DECODED SIGNAL FOR SEVERAL COMBINATIONS OF TI DURING A POSSIBLE BRUTE FORCE ATTACK.

\begin{tabular}{|c|c|}
\hline Combination & $\boldsymbol{B E \boldsymbol { R } _ { \boldsymbol { d } }}$ \\
\hline$\tau_{l}=\tau_{2}=\tau_{3}=\tau_{4}=\tau_{5}=\tau_{6}=\tau_{7}=0 ;$ & $36 \%$ \\
\hline$\tau_{l}=\tau_{2}=\tau_{3}=\tau_{5}=\tau_{6}=\tau_{7}=0 ; \tau_{4}$ correct & $36 \%$ \\
\hline$\tau_{l}=\tau_{2}=\tau_{5}=\tau_{6}=\tau_{7}=0 ; \tau_{3}$ and $\tau_{4}$ correct & $13 \%$ \\
\hline$\tau_{l}=\tau_{2}=\tau_{6}=\tau_{7}=0 ; \tau_{3}, \tau_{4}$ and $\tau_{5}$ correct & $2.710^{-13}$ \\
\hline$\tau_{l}=\tau_{6}=\tau_{7}=0 ; \tau_{2}, \tau_{3}, \tau_{4}$ and $\tau_{5}$ correct & $2.710^{-13}$ \\
\hline$\tau_{l}=\tau_{7}=0 ; \tau_{2}, \tau_{3}, \tau_{4}, \tau_{5}$ and $\tau_{6}$ correct & $<1.010^{-15}$ \\
\hline
\end{tabular}

In principle, $\tau_{i}$ is a continuous random variable. However, if the delay used by the eavesdropper, $\tau_{i}^{\prime}$ ( $i=1$ to $n$ ), is in the range $\tau_{i^{-}} \Delta \tau_{i} \leq \tau_{i}^{\prime} \leq \tau_{i}+\Delta \tau_{i}$ and $\Delta \tau_{i}$ is small enough, the attack may still be prosperous. Computationally, it is hard to determine a minimum value for $\Delta \tau_{i}$ because this requires the use of very high sample rates, $f_{S}$. In fact our simulations have shown that reliable results demand the product $\left(f_{S} \Delta \tau_{i}\right)$ to be higher than 40 (i.e., there must be at least 40 sampling points in the interval $\Delta \tau_{i}$ ). Within our presently available computational power, we found that $\Delta \tau_{i}=1$ ps leads to $B E R_{d} \sim 3 \%$. We will consider this $\Delta \tau_{i, \max }=1 \mathrm{ps}$ limit as a very conservative estimate for $\Delta \tau_{i}$.

Assuming that $\tau_{\max }=10 T_{b}=250 \mathrm{ps}$ is, in a pessimistic scenario for security, known and considering $\Delta \tau_{i, \max }=1 \mathrm{ps}$ shows that the brute force attack may require 250 tries per spectral slice to be effective. Since Table I shows that $\tau_{i}^{\prime}$ must be properly known for at least three spectral slices, then the unauthorized user may need up to $250 ! /(247 !) \sim 1.510^{7}$ attempts to perform a successful attack.

This is also a very conservative estimate because, in practice, the encoded signal would be possibly captured inside the TON and would then be propagated to the unauthorized receiver facilities. For this reason, noise and other physical impairments accumulated through the propagation would corrupt the encoded signal whose quality would be worse than that considered in Table I (back-to-back case). To compensate these deleterious effects, the eavesdropper could either work with a shorter $\Delta \tau_{i}$, or deal with a higher number of spectral slices. In both of these situations, the number of attempts necessary for achieving a prosperous attack would increase. Fig. 18 shows the number of necessary brute force attacks, for $\tau_{m a x}=10 T_{b}$ and $R_{b}=40 \mathrm{Gbps}$, as a function $\Delta \tau_{i}$ and the number of considered slices, $n_{s}$, necessary to decode the optical signal. It is seen that if $n_{s}$ needs to be increased by only one, the number of necessary attempts to decode the signal raises by at least two orders of magnitude. A decrease from 1 ps to $50 \mathrm{fs}$ in $\Delta \tau_{i}$ also implies an increase of two orders of magnitude in the number of necessary attempts when $n_{s}=3$, and of eight orders of magnitude when $n_{s}=7$. 


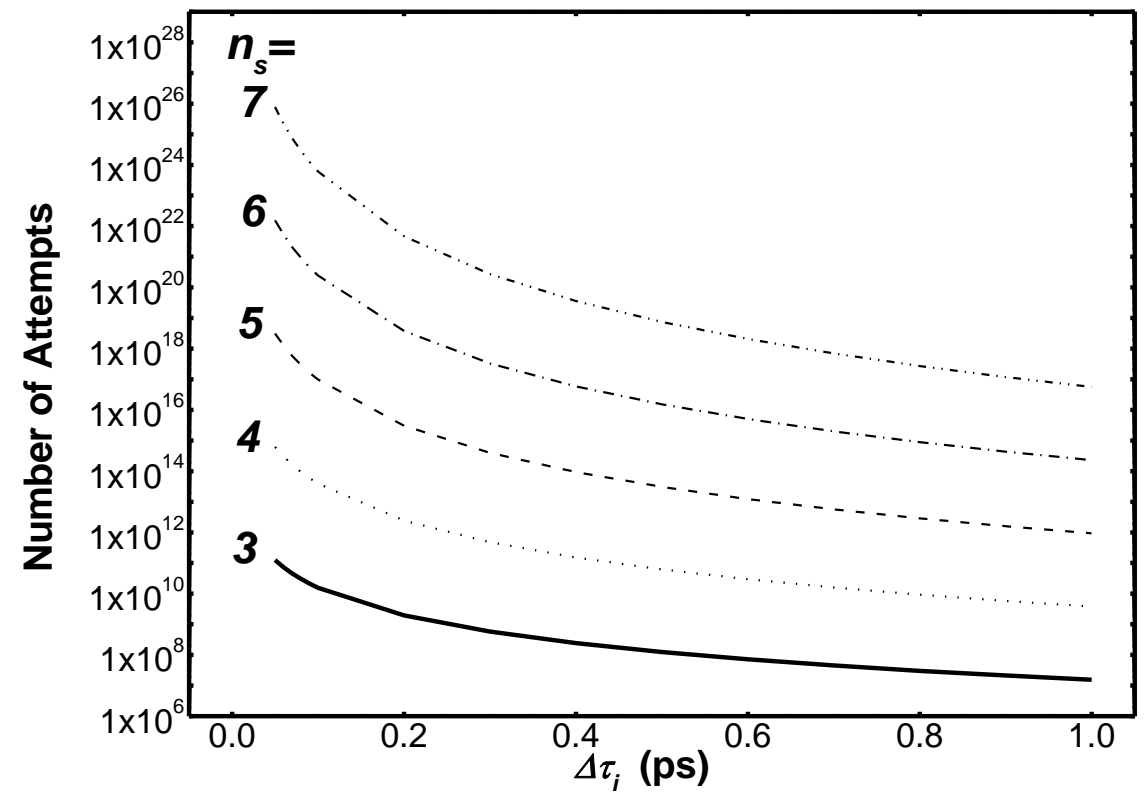

Fig. 18. Dependence between the maximum number of brute force attack attempts necessary for decoding an encoded NRZ-OOK signal and $\Delta \tau_{i}$, for different values of $n_{s}$.

\section{CONCLUSION}

We have systematically analyzed the performance of a new two-stage all-optical cryptography technique that is bit-rate independent and transparent to modulation formats. It was shown that the first of these stages, related to spectral amplitude encoding, is essential to increase the BER of encoded signals to desired elevated levels. In fact, our results show that $B E R_{E}$ as high as 45 and $28 \%$ are attainable, respectively, for input NRZ-OOK and DQPSK signals. The second stage performs spectral delay encoding and its use is mandatory to promote temporal signal scrambling and to increase the degree of security against attacks based on waveform analysis. At the receiver side, our results indicate that the considered signals may be properly decoded after propagation distances comparable to those expected for metropolitan area networks. These results suggest that the technique may have a very good practical performance and serve as a good foundation for future experimental research.

Since the investigated technique relies solely on linear devices and effects, its implementation is much simpler and advantageous in relation to other presented in literature [14- 16]. Moreover, the use of spectral delay encoding also avoids, to certain extent, the presence of OBP that happens in [13] and constitute another upside for the technique considered here.

Although our analysis was not focused on any particular technology, the approached technique could be implemented either with discrete components or in integrated optics. In the first of these approaches, the encoder and decoder could be implemented by properly connecting specially designed optical multiplexers, optical attenuators, and optical delay lines. Alternatively, a basic 4-f setup [18] whose amplitude and delays are controlled by a spatial light modulator (SLM) could be utilized as 
well. Silicon photonics is suitable for deploying the considered devices in an integrated optics strategy. In all of these cases, special attention should be given to the influence of temperature over the frequency position of OBPFs and other optical devices.

Finally, we remark that the technique could also be extended to a dynamic approach, where the cryptographic key varies with time. In this situation, the encoder/ decoder attenuators could be substituted by optical modulators. As is the case for most of encrypting technologies dynamic keys are of interest because they increase the degree of communications security.

\section{ACKNOWLEDGMENT}

Authors thank CNPq and FAPESP for funding this work in the scope of Fotonicom Program, under grants 574017/2008-9, 08/57857-2, 310644/2011-9, and 310990/2011-4. Authors also thank Prof. Hugo L. Fragnito and Prof. Ben-Hur V. Borges for valuable discussions. Finally, authors thank VPIphotonics ${ }^{\mathrm{TM}}$ for providing academic licenses of the simulation software utilized in this work.

\section{REFERENCES}

[1] K. Kitayama, M. Sasaki, S. Araki, M. Tsubokawa, A. Tomita, K. Inoue, K. Harasawa, Y. Nagasako, and A. Takada, "Security in Photonic Networks: Threats and Security Enhancement," IEEE/OSA Journal of Lightwave Technology, vol. 29, pp. 3210-3222, November, 2011.

[2] K. Shaneman, and S. Gray, "Optical network security: technical analysis of fiber tapping mechanisms and methods for detection \& prevention," IEEE Military Communications Conference, vol. 2, pp. 711-716, 2004.

[3] N. Skorin-Kapov, J. Chen, and L. Wosinska, "A New Approach to Optical Networks Security: Attack-Aware Routing and Wavelength Assignment," IEEE/ACM Transactions on Networking, vol. 18, pp. 750-760, June 2010.

[4] L. Zhang, X. Xin, B. Liu, and Y. Wang, "Secure OFDM-PON Based on Chaos Scrambling," IEEE Photonics Technology Letters, vol. 23, pp. 998-1000, July 2011.

[5] P. Juleang, R. Putthacharoen, S. Mitatha, and P.P. Yupapin, "Highly secured optical communication by optical key and identification address," Elsevier Optik - International Journal for Light and Electron Optics, vol. 124, pp. 834-839, May 2013.

[6] A. Argyris, D. Syvridis, L. Larger, V. Annovazzi-Lodi, P. Colet, I. Fischer, J. García-Ojalvo, C.R. Mirasso, L. Pesquera, and K.A. Shore, "Chaos-based communications at high bit rates using commercial fibre-optic links," Nature 438, pp. 343-346, 2005.

[7] Y. Hong, M.W. Lee, J. Paul, P.S. Spencer, and K.A. Shore, "GHz Bandwidth Message Transmission Using Chaotic Vertical-Cavity Surface-Emitting Lasers,” IEEE/OSA Journal of Lightwave Technology, vol. 27, pp. 5099-5105, november 2009.

[8] J-P. Goedgebuer, P. Levy, L. Larger, C-C. Chen, and W.T. Rhodes, "Optical communication with synchronized hyperchaos generated electrooptically," IEEE Journal of Quantum Electronics, vol. 38, pp. 1178-1183, September 2002.

[9] N. Kostinski, K. Kravtsov, and P.R. Prucnal, "Demonstration of an All-Optical OCDMA Encryption and Decryption System With Variable Two-Code Keying," IEEE Photonics Technology Letters, vol. 20, pp. 2045-2047, December 2008.

[10] T.H. Shake, "Security performance of optical CDMA Against eavesdropping," IEEE/OSA Journal of Lightwave Technology, vol. 23, pp. 655-670, February 2005.

[11] C-C. Yang, "Hybrid Wavelength-Division-Multiplexing/Spectral-Amplitude-Coding Optical CDMA System," IEEE Photonics Technology Letters, vol. 17, pp. 1343-1345, june 2005.

[12] K. Kitayama, X. Wang, and N. Wada, "OCDMA Over WDM PON-Solution Path to Gigabit-Symmetric FTTH,” IEEE/OSA Journal of Lightwave Technology, vol. 24, pp. 1654-1662, 2006

[13] J.A. Cornejo, C.E. Perez, and J-L. B. Tocnaye, "Non-invasive WDM channel scrambling for secure high data rate optical transmissions," IEEE/OSA Journal of Lightwave Technology, vol. 25, pp. 2081-2089, 2007.

[14] L. Yi, T. Zhang, Z. Li, J. Zhou, Y. Dong, W. Hu, "Secure optical communication using stimulated Brillouin scattering in optical fiber," Elsevier Optics Communications, vol. 290, pp. 146-151, March 2013.

[15] S. Singh, Lovkesh, X. Ye, and R.S. Kaler, "Design of Ultrafast Encryption and Decryption Circuits for Secured Optical Networks," IEEE Journal of Quantum Electronics, vol. 48, pp. 1547-1553, 2012.

[16] K. Mukherjee, "A method of implementation of frequency encoded all optical encryption decryption using four wave mixing," Elsevier Optik - International Journal for Light and Electron Optics, vol. 122, pp. 1407-1411, 2011.

[17] M.L.F. Abbade, L.A. Fossaluzza Jr., R.F. Silva, and E.A.M. Fagotto, "Criptografia Óptica Mediante Controle Analógico da Amplitude e do Atraso de Fatias Espectrais: Análise para Sinais NRZ,” (in Portuguese) MOMAG, 2012.

[18] M. Kavehrad, Fellow, and D. Zaccarh, "Optical Code-Division-Multiplexed Systems Based on Spectral Encoding of Noncoherent Sources," Journal of Lightwave Technology, vol. 13, pp. 534-545, 1995. B.P. Lathi, "Modern Digital and Analog Communication Systems," $3^{\text {rd }}$ Edition, Oxford Press, 1998.

[19] B.P. Lathi, "Modern Digital and Analog Communication Systems," $3^{\text {rd }}$ Edition, Oxford Press, 1998.

[20] R.F. Silva, "Análise de criptografia óptica realizada mediante controle da amplitude e do atraso de fatias espectrais geradas com perfil de filtros ópticos comerciais," (in Portuguese) Master Dissertation, PUC-Campinas, 2012. 